\title{
Calibração e avaliação do modelo CROPGRO para a cultura do feijão caupi no Recôncavo Baiano
}

\author{
Aristides F. Lima Filho', Mauricio A. Coelho Filho² \& Alexandre B. Heinemann ${ }^{3}$
}

\begin{abstract}
RESUMO
Objetivou-se, com este trabalho, calibrar e avaliar o modelo CROPGRO para a cultura do feijão caupi nas condições edafoclimáticas do Recôncavo da Bahia. Foram instalados cinco experimentos em campo, em diferentes épocas de semeadura: março de 2007, janeiro de 2010, maio de 2010, outubro de 2010 e julho de 2011. Na calibração utilizou-se o cultivo de maior produtividade. Para a validação do modelo o índice de área foliar máximo foi subestimado em 6,8\% e a diferença entre as produtividades simulada e observada foi de $1 \%$. Na avaliação foram utilizados os demais experimentos sendo as diferenças máximas entre dados observados e simulados de sete dias para as fases fenológicas e as diferenças de produtividade se situaram entre -30,8\% (experimento com déficit hídrico) e 13,4\% (experimento do período de chuvas). De acordo com os resultados conclui-se que o modelo simula satisfatoriamente o crescimento e o desenvolvimento do caupi no Recôncavo Baiano, em condições ótimas, apresentando limitações em simulações com situações de déficit hídrico.
\end{abstract}

Palavras-chave: modelagem, Vigna unguiculata, DSSAT

\section{Calibration and evaluation of CROPGRO model for cowpea in Recôncavo of Bahia - Brazil}

\begin{abstract}
This study aimed to calibrate and evaluate the crop model CROPGRO-cowpea for Recôncavo of Bahia. Five field experiments were implemented at different sowing dates: March 2007, January 2010, May 2010, October 2010 and July 2011. For crop model calibration the experiment with the highest yield was used. For calibration the maximum leaf area index was underestimated by $6.8 \%$ and the difference between simulated and observed yield was only $1 \%$. For crop model evaluation results of other field experiments were used and the crop model showed a maximum difference of 7 days between observed and simulated phenological dates. For observed and simulated yields there were differences of $30.8 \%$ for water deficit experiment and $13.4 \%$ for experiment with no water deficit. Based on that it is possible to conclude that the crop model simulates the growth and development of cowpea in Recôncavo of Bahia well for no water deficit condition and shows limitations under water deficit condition.
\end{abstract}

Key words: modeling, Vigna unguiculata, DSSAT

\footnotetext{
1 Instituto Federal de Educação, Ciência e Tecnologia da Bahia, CEP 40300-010, Salvador, BA. Fone: (71) 2102-9536. E-mail: aristides@ifba.edu.br

${ }^{2}$ Laboratório de Irrigação e Fertirrigação/Embrapa Mandioca e Fruticultura Tropical. CEP 44380-000, Cruz das Almas, BA. Fone: (75) 3621-8021. E-mail: macoelho@cnpmf.embrapa.br

${ }^{3}$ Embrapa Arroz e Feijão, Rodovia GO 462, km 12, Zona Rural, CEP 75375-000, Santo Antônio de Goiás, GO. E-mail: alexbh@cnpaf.embrapa.br
} 


\section{INTRODUÇÃO}

O feijão caupi (Vigna unguiculata L. Walp.) é uma das culturas mais importantes para as regiões Norte e Nordeste do Brasil, significando alimento de alto conteúdo proteico, além de gerar emprego e renda para as famílias de baixo poder aquisitivo que vivem nessas regiões. A produtividade, entretanto é muito baixa.

A utilização de técnicas de cultivo, a adubação e a irrigação, podem ser utilizadas para o incremento da produção, porém a maioria das novas tecnologias demanda tempo e recursos, muitas vezes utilizados somente para culturas nobres.

A utilização de modelos que simulem o crescimento e o desenvolvimento de culturas é uma alternativa importante para estudos de culturas de importância comercial.

Os modelos também permitem estudos relativos ao impacto das mudanças climáticas e quantificação das interações do genótipo com o ambiente e com o manejo, particularmente em situações com variabilidade climática (Heinemann et al., 2008; 2009).

Segundo Muniz et al. (2007) os procedimentos de modelagem facilitam a integração de conceitos científicos e resultados experimentais, sendo capazes de melhorar o entendimento da dinâmica de uma lavoura. Esses modelos também podem ser usados para determinar e mensurar o impacto de diferentes estratégias de alocação de recursos sobre os resultados produtivos e econômicos.

De acordo com Lorençoni et al. (2010) os modelos podem ser utilizados para integrar os conhecimentos dos processos biofísicos que regem o sistema solo-água-planta, permitindo identificar e avaliar as incertezas na produção, associadas às diferentes opções de manejo.

Dentre os vários modelos de crescimento de culturas disponíveis na literatura se destacam os incluídos no sistema computacional DSSAT (Decision Support System for Agrotechnology Transfer). Este sistema tem sido utilizado por muitos pesquisadores nas mais diversas condições climáticas e de solo, para obtenção de informações que auxiliam no planejamento e no manejo agrícola (Hoogenboom et al., 2004).

No Nordeste do Brasil, Bastos (1999) trabalhou com o modelo CROPGRO (DSSAT) adaptando-o para simulação do crescimento e do desenvolvimento do caupi no Estado do Piauí, obtendo bons resultados para estimativa de biomassa e índice de área foliar.

Foram realizados trabalhos em outros locais como Viçosa, Minas Gerais, com o mesmo modelo (Oliveira et al., 2012), como ferramenta de previsão de rendimento de feijão (Phaseolus vulgaris L.), e em Maringá, estado do Paraná, Dallacort et al. (2010) utilizaram o referido modelo para determinação das melhores épocas de semeadura da cultura do feijão, encontrando bons resultados.

Deve-se ressaltar que os modelos utilizados na tentativa de simular uma cultura em campo, são aproximações da realidade e servem para nortear a tomada de decisões, além de terem aplicação restrita a condições de clima, solo e planta. Ciente dessas limitações o objetivo deste trabalho foi calibrar e avaliar o modelo CROPGRO, para a cultura do feijão caupi nas condições edafoclimáticas do Recôncavo Baiano.

\section{Material e Mètodos}

Os experimentos foram montados na área experimental da Embrapa Mandioca e Fruticultura, em Cruz das Almas, BA, cujas coordenadas geográficas são: $12^{\circ} 40^{\prime} 39^{\prime \prime} \mathrm{S}$ e $39^{\circ}$ $06^{\prime} 23^{\prime}$ 'W e a altitude é de $225 \mathrm{~m}$. O clima é tropical quente e úmido, com pluviosidade média anual de $1.170 \mathrm{~mm}$ e variações entre 900 e $1300 \mathrm{~mm}$, com o período chuvoso compreendido entre março e agosto e o seco entre setembro e fevereiro. A temperatura média anual é de $24,5{ }^{\circ} \mathrm{C}$ e a umidade relativa é de $80 \%$.

A cultura utilizada foi o feijão caupi (Vigna unguiculata $\mathrm{L}$. (WALP.)), cultivar BRS Guariba, cujo hábito de crescimento, indeterminado, apresenta porte semiereto, entra em floração plena aos 41 dias e tem ciclo entre 65 e 70 dias.

O solo da área experimental é classificado como Latossolo Amarelo Distrófico Típico, textura franco argilo-arenosa (Tabela 1).

As correções e adubações foram realizadas de acordo com a análise química do solo (Tabelas 2 e 3 ) e baseadas na recomendação para a cultura. Foram realizadas capinas para controle das plantas daninhas e se aplicou formicida à base de sulfuramida.

Tabela 1. Análise física do solo da área experimental em Cruz das Almas

\begin{tabular}{|c|c|c|c|c|c|c|c|c|}
\hline \multirow{2}{*}{$\begin{array}{l}\text { Profundidade } \\
\text { (m) }\end{array}$} & Areia & Silte & Argila & \multirow{2}{*}{\multicolumn{2}{|c|}{ US $\left(\mathrm{kg} \mathrm{dm}^{-3}\right)$}} & CC & PM & AD \\
\hline & & $(\%)$ & & & & \multicolumn{3}{|c|}{$\left(m^{3} \mathrm{~m}^{-3}\right)$} \\
\hline $0-0,2$ & 73,2 & 8,7 & 18,1 & 1,67 & 2,53 & 0,1761 & 0,0980 & 0,0781 \\
\hline $0,2-0,4$ & 62,9 & 6,8 & 30,3 & 1,66 & 2,50 & 0,1936 & 0,1514 & 0,0422 \\
\hline $0,4-0,7$ & 60,0 & 7,7 & 32,3 & 1,43 & 2,46 & 0,1837 & 0,1320 & 0,0517 \\
\hline $0,7-1,0$ & 53,3 & 8,1 & 38,4 & 1,39 & 2,40 & 0,2039 & 0,1435 & 0,0604 \\
\hline
\end{tabular}

Tabela 2. Análise química do solo da área dos experimentos de janeiro de 2010 e maio de 2010

\begin{tabular}{|c|c|c|c|c|c|c|c|c|c|c|c|c|}
\hline \multirow{2}{*}{$\mathrm{pH}$} & \multirow{2}{*}{$\begin{array}{l}\text { M.O. } \\
\left(g^{2} g^{-1}\right)\end{array}$} & $\mathbf{P}$ & K & $\mathrm{Ca}+\mathrm{Mg}$ & $\mathrm{Ca}$ & $\mathrm{Mg}$ & Al & $\mathrm{H}+\mathbf{A l}$ & $\mathrm{Na}$ & $S$ & CTC & V \\
\hline & & \multicolumn{2}{|c|}{$\left(\mathrm{mg} \mathrm{dm}{ }^{-3}\right)$} & \multicolumn{8}{|c|}{$\left(\mathrm{cmol}_{\mathrm{c}} \mathrm{dm}^{-3}\right)$} & $(\%)$ \\
\hline
\end{tabular}

Tabela 3. Análise química do solo da área dos experimentos de maio e outubro de 2010 e julho de 2011

\begin{tabular}{|c|c|c|c|c|c|c|c|c|c|c|c|c|}
\hline \multirow{2}{*}{$\mathrm{pH}$} & \multirow{2}{*}{$\begin{array}{l}\text { M.O. } \\
\left(g^{-1}\right)\end{array}$} & $\mathbf{P}$ & K & $\mathrm{Ca}+\mathrm{Mg}$ & $\mathrm{Ca}$ & Mg & Al & $\mathrm{H}+\mathrm{Al}$ & $\mathrm{Na}$ & $S$ & CTC & V \\
\hline & & \multicolumn{2}{|c|}{$\left(\mathrm{mg} \mathrm{dm}^{-3}\right)$} & \multicolumn{8}{|c|}{$\left(\mathrm{cmol}_{\mathrm{c}} \mathrm{dm}^{-3}\right)$} & $(\%)$ \\
\hline 6,21 & 1,21 & 14 & 47 & 3,6 & 2,2 & 1,4 & 0,05 & 1,37 & 0,12 & 3,84 & 5,21 & 73,70 \\
\hline
\end{tabular}


O trabalho de campo foi desenvolvido em cinco etapas, variando as épocas de semeadura. $\mathrm{O}$ experimento $\mathrm{P}_{\mathrm{MR} 07}$ (semeado em 21/03/2007) foi implantado em uma área de $12 \times 12 \mathrm{~m}$ com espaçamento entre covas de 0,30 por $0,50 \mathrm{~m}$, colocando-se cinco sementes por cova e se deixando três plantas por cova, após o desbaste. O manejo da água foi feito com TDR (reflectometria no domínio de tempo), repondo-se a água de acordo com a variação da umidade do solo.

Nos cultivos de janeiro (semeados em 20/01/2010) o manejo da água também foi feito com TDR, aplicando-se restrições de $0,25,50,75$ e $100 \%$ da capacidade de campo, respectivamente para os cultivos $\mathrm{P}_{\mathrm{J} 0}, \mathrm{P}_{\mathrm{J} 25}, \mathrm{P}_{\mathrm{J} 50}, \mathrm{P}_{\mathrm{J} 75}$ e $\mathrm{P}_{\mathrm{J} 100}$, no início da floração. As parcelas foram de $8 \times 6 \mathrm{~m}$ com espaçamento entre covas de 0,20 por $0,70 \mathrm{~m}$, semeando-se três sementes por cova e se deixando uma planta por cova, após o desbaste.

$\mathrm{O}$ cultivo de maio $\left(\mathrm{P}_{\text {MAI10 }}\right)$, semeado em 15/05/2010, foi realizado numa área de $27 \times 17 \mathrm{~m}$, o espaçamento entre covas foi de 0,20 por $0,70 \mathrm{~m}$, semeando-se três sementes por cova e se deixando uma planta por cova após, o desbaste. A umidade do solo foi monitorada com sonda de capacitância de modelo Diviner 2000.

Para os cultivos de outubro $\left(\mathrm{P}_{\mathrm{OI}}\right.$ e $\left.\mathrm{P}_{\mathrm{ONI}}\right)$, semeados em $01 / 10 / 2010$, foi montado um experimento inteiramente casualizado com dois tratamentos (doze parcelas irrigadas e doze não irrigadas), a fim de se verificar o efeito da irrigação na produtividade da cultura nesta época. Utilizou-se o sistema de irrigação localizada por gotejamento, trabalhando-se com gotejadores com vazão de $4 \mathrm{~L} \mathrm{~h}^{-1}$, espaçados $0,40 \mathrm{~m}$. O tamanho das parcelas foi de $3,0 \times 5,0 \mathrm{~m}$, com espaçamento entre covas de 0,20 por $0,70 \mathrm{~m}$, semeando-se três sementes por cova e se deixando uma planta por cova, após o desbaste. O manejo da água foi feito com sonda de capacitância (modelo Diviner 2000), repondo-se a água de acordo com a variação da umidade do solo. Semanalmente, uma planta por parcela foi coletada para determinação da matéria seca e da área foliar.

O cultivo $P_{\text {IUL11 }}$, semeado em 27/07/2011, foi implantado em uma área de $27 \times 17 \mathrm{~m}$, com espaçamento entre covas de 0,20 por $0,70 \mathrm{~m}$, semeando-se três sementes por cova e se deixando uma planta por cova, após o desbaste. A umidade do solo foi monitorada com sonda de capacitância.

Com exceção dos cultivos de outubro $\left(\mathrm{P}_{\mathrm{OI}}\right.$ e $\left.\mathrm{P}_{\mathrm{ONI}}\right)$, foram coletadas três subamostras de plantas semanalmente, com três plantas cada uma, a partir dos vinte dias após a semeadura, para determinação da matéria seca $\left(\mathrm{g}_{\text {planta }}{ }^{-1}\right)$ e da área foliar $\left(\mathrm{m}^{2}\right)$, sendo que a matéria seca total resultou da soma da matéria seca das diversas partes (raiz, haste, folhas e vagens), após secarem em estufa de ventilação forçada $\left(65 \pm 5^{\circ} \mathrm{C}\right)$, até atingirem massa constante.

A área foliar foi determinada antes das amostras serem levadas à estufa, conforme Lima et al. (2008) utilizando a Eq. 1:

$$
\mathrm{AFT}=\sum_{\mathrm{i}}^{\mathrm{n}} 0,9915(\text { C.L })^{0,9134}
$$

em que:

$$
\begin{array}{ll}
\text { AFT } & \text { - área foliar total, } \mathrm{m}^{2} \\
\Sigma & \text { - somatório das folhas } \\
\text { C } & \text { - comprimento da folha, } \mathrm{m} \\
\text { L } & \text { - largura da folha, } \mathrm{m}
\end{array}
$$

Foram observadas e anotadas as datas de semeadura, emergência, floração (50\% das plantas com flores), datas do aparecimento da primeira vagem, do aparecimento do primeiro grão e maturidade fisiológica. Também foram determinadas as produtividades médias de grãos $\left(\mathrm{kg} \mathrm{ha}^{-1}\right)$ corrigidas para $0 \% \mathrm{de}$ umidade possibilitando a comparação com os dados simulados pelo modelo.

O teor de água das sementes foi determinado pelo método da estufa utilizando-se duas amostras de 50 sementes, as quais foram pesadas em balança com precisão de $0,01 \mathrm{~g}$ para obtenção da massa úmida e posteriormente foram colocadas para secar em estufa a $105 \pm 3{ }^{\circ} \mathrm{C}$, por $24 \mathrm{~h}$ (Brasil, 2009). Após as $24 \mathrm{~h}$ realizou-se nova pesagem para obtenção da massa seca.

A calibração do modelo foi feita de acordo com Bastos (1999) ajustando-se as datas das fases fenológicas (florescimento, aparecimento da primeira vagem, aparecimento do primeiro grão e maturidade fisiológica) para posteriormente se proceder aos ajustes de matéria seca, índice de área foliar e produtividade.

\section{Resultados e Discussão}

O cultivo $\mathrm{P}_{\mathrm{MAR} 07}$, devido ao período favorável de chuvas, recebeu apenas $15,6 \mathrm{~mm}$ de água de irrigação na floração para complementar as necessidades de água. $\mathrm{O}$ total aplicado foi de $307,6 \mathrm{~mm}$. Os cultivos de janeiro $\left(\mathrm{P}_{\mathrm{J} 0}, \mathrm{P}_{\mathrm{J} 25}, \mathrm{P}_{\mathrm{J} 50}, \mathrm{P}_{\mathrm{J} 75}\right.$ e $\left.\mathrm{P}_{\mathrm{J} 100}\right)$ foram planejados para receber déficits diferenciados de água, porém ocorreu precipitação de $102 \mathrm{~mm}$ entre o aparecimento da primeira flor e a colheita, mascarando o efeito dos déficits. O cultivo $\mathrm{P}_{\mathrm{J} 50}$ foi descartado devido a erros na coleta de dados

\begin{tabular}{|c|c|c|c|c|c|c|c|c|c|c|}
\hline Cultivo & $\frac{P}{S-E}$ & $\frac{\text { I }}{\text { S-E }}$ & $\begin{array}{c}\text { Total } \\
\text { S-F }\end{array}$ & $\frac{P}{E-F}$ & $\frac{\text { I }}{E-F}$ & $\begin{array}{c}\text { Total } \\
\text { E-F }\end{array}$ & $\frac{P}{F-C}$ & $\frac{\text { I }}{\text { F-C }}$ & $\begin{array}{c}\text { Total } \\
\text { F-C }\end{array}$ & Total \\
\hline$P_{\mathrm{JO}}$ & 2,6 & 30,0 & 32,6 & 37,2 & 95,6 & 132,8 & 102,0 & 0,0 & 102,0 & 267,4 \\
\hline$P_{\mathrm{J} 75}$ & 2,6 & 30,0 & 32,6 & 37,2 & 129,3 & 166,5 & 102,0 & 31,6 & 133,6 & 332,7 \\
\hline$P_{J 100}$ & 2,6 & 30,0 & 32,6 & 37,2 & 140,5 & 177,7 & 102,0 & 42,1 & 144,1 & 354,4 \\
\hline $\mathrm{P}_{\text {MAIO }}$ & 20,2 & 0,0 & 20,2 & 61,9 & 0,0 & 61,9 & 290,1 & 0,0 & 290,1 & 372,2 \\
\hline $\mathrm{P}_{\mathrm{JUL11}}$ & 24,0 & 0,0 & 24,0 & 127,0 & 0,0 & 127,0 & 83,2 & 0,0 & 83,2 & 234,2 \\
\hline
\end{tabular}
de campo. O total de água aplicada foi, em média, $310,9 \mathrm{~mm}$ para os cultivos de janeiro (Tabela 4).

Tabela 4. Lâmina total de água aplicada $(\mathrm{mm})$ nas diferentes fases fenológicas do caupi em Cruz das Almas em 2007,2010 e 2011

S - Semeadura; E - Emergência; F - Floração; C - Colheita; P - Precipitação (mm); I - Irrigação (mm) 
O Cultivo $\mathrm{P}_{\text {MAII0 }}$, conduzido na época das chuvas, recebeu o total de $372,2 \mathrm{~mm}$ sendo que $290,1 \mathrm{~mm}$ (cerca de $78 \%$ ) ocorreram entre a floração e a colheita, dando uma média de $8,8 \mathrm{~mm} \mathrm{~d}^{-1}$, o que representa uma grande quantidade de água, provavelmente muito acima das necessidades das plantas, podendo influenciar negativamente na produtividade apesar do excelente desempenho na fase vegetativa observado em campo. Bastos et al. (2008) encontraram um consumo máximo de $5,4 \mathrm{~mm} \mathrm{~d}^{-1}$ para o caupi na fase de maior demanda (período reprodutivo).

No cultivo $\mathrm{P}_{\mathrm{OI}}$ constatou-se precipitação pluviométrica de $81,1 \mathrm{~mm}$ além de irrigação complementar de $43,6 \mathrm{~mm}$, totalizando $124,7 \mathrm{~mm}$ desde a fase de emergência até o início da floração, o que dá uma média diária de $3,7 \mathrm{~mm}$. Da floração à colheita foi aplicado o total de 78,2 $\mathrm{mm}$ (sendo $4,8 \mathrm{~mm}$ de precipitação pluvial e 73,4 mm de irrigação), totalizando 232,1 $\mathrm{mm}$.

O cultivo $\mathrm{P}_{\mathrm{ONI}}$ de outubro recebeu $81,1 \mathrm{~mm}$ de precipitação pluviométrica da emergência ao início da floração, dando uma média diária baixa $(2,4 \mathrm{~mm})$ e apenas $4,8 \mathrm{~mm}$ entre o aparecimento da primeira flor e a colheita. Esta baixa quantidade de água influenciou, provavelmente, a produtividade, de vez que, segundo Sousa et al. (2009) o efeito do estresse hídrico em feijoeiro causa reduções nos componentes de produção e é mais severo quando ocorre nas fases de floração e frutificação.

No cultivo $\mathrm{P}_{\text {JUL11 }}$, conduzido sem irrigação, observa-se que a distribuição de água de chuva ao longo do ciclo das plantas foi muito similar à aplicação de água via irrigação no cultivo $\mathrm{P}_{\mathrm{O}}$, assim como o total de água aplicada em ambos foi próxima, 234,2 $\mathrm{mm}$ e 232,1 $\mathrm{mm}$ respectivamente para $\mathrm{P}_{\text {JUL11 }} \mathrm{e}$ $\mathrm{P}_{\mathrm{OI}}$, proporcionando bom desenvolvimento das plantas.

Os coeficientes ajustados na calibração foram: área foliar específica $\left(255 \mathrm{~cm}^{2} \mathrm{~g}^{-1}\right.$, área máxima de trifólio $\left(40 \mathrm{~cm}^{2}\right)$, peso máximo do grão $(0,17 \mathrm{~g}$, duração do período de enchimento do grão (16 dias), número médio de grãos por vagem $(13,2)$, tempo pata atingir o final do crescimento da vagem (14 dias), tempo entre semeadura e emergência (5 dias), tempo entre emergência e primeira folha verdadeira (5 dias), tempo entre maturidade fisiológica e colheita (5 dias), peso específico da folha $\left(0,0039 \mathrm{~g} \mathrm{~cm}^{-2}\right)$

As temperaturas máximas e mínimas do ar se mantiveram dentro da faixa ideal para o caupi que, segundo Araújo \& Watt (1988) devem estar entre 20 e $35^{\circ} \mathrm{C}$ (Figura 1).

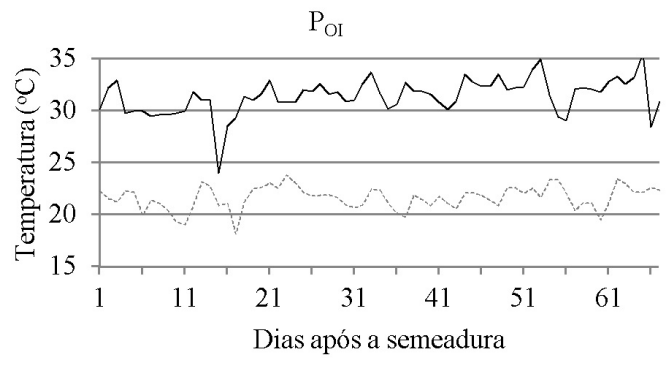

Figura 1. Temperaturas máximas e mínimas no cultivo $P_{O}$

Os resultados da calibração com os coeficientes ajustados para as fases fenológicas se encontram na Tabela 5. Para o aparecimento da primeira flor (44 DAS), aparecimento da primeira vagem (47 DAS), do primeiro grão (51 DAS) e maturidade fisiológica (68 DAS) observou-se perfeita coincidência dos dados simulados com os dados de campo.

Tabela 5. Resultado da calibração do modelo CROPGRO para o feijão caupi (cultivo $\mathrm{P}_{\mathrm{OI}}$ )

\begin{tabular}{lccc}
\hline \multicolumn{1}{c}{ Variável } & Simulado & Observado & Diferença \\
Primeira flor (DAS) & 44 & 44 & 0 \\
Primeira vagem (DAS) & 47 & 47 & 0 \\
Primeiro grão (DAS) & 51 & 51 & 0 \\
Maturidade fisiológica (DAS) & 68 & 68 & 0 \\
\hline
\end{tabular}

Observou-se boa proximidade entre os valores obtidos em campo e os dados simulados, conforme explicitado pelos indicadores de desempenho sendo alto o coeficiente de determinação $\left(\mathrm{R}^{2}\right.$ de 0,982$)$ e de concordância de Willmott (dW de 0,969$)$ e relativamente baixo o erro absoluto médio (EAM) de $-311,0 \mathrm{~kg} \mathrm{ha}^{-1}$ (Figura 2A). O máximo de matéria seca observada ( $\left.3.043 \mathrm{~kg} \mathrm{ha}^{-1}\right)$ foi alcançado aos 68 dias após a semeadura. Valores esses compatíveis com os encontrados por Bastos (1999) que obteve coeficientes de determinação variando de 0,77 a 0,98 , erros absolutos médios variando de 204 a $325 \mathrm{~kg} \mathrm{ha}^{-1}$ e pico de matéria seca aos 63 dias após a semeadura, trabalhando no Piauí com a mesma cultura porém com cultivares diferentes.

Percebeu-se também um aumento lento da matéria seca no início da fase vegetativa, crescendo exponencialmente posteriormente por ocasião da emissão das vagens. Fato também relatado por Bastos (1999) em caupi e Cruz et al. (2010) em soja.

As estimativas de IAF obtidas com o modelo se aproximaram dos dados de campo (Figura 2B), demonstrando boa precisão nesta fase de calibração e apresentando diferenças que variaram de 0,04 a 0,74 . O IAF máximo simulado foi de 2,46 aos 52 DAS enquanto o IAF máximo observado foi de 2,64 aos

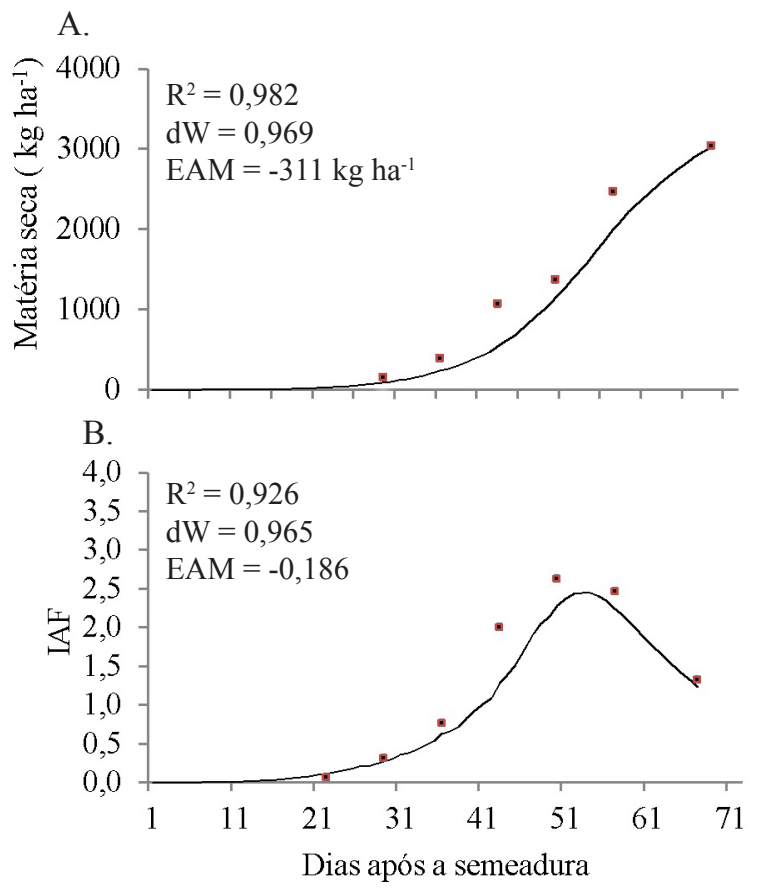

Figura 2. Estimativa de matéria seca em (A) e de índice de área foliar (IAF) em (B) na fase de calibração 
49 DAS, dando uma diferença de $6,8 \%$. Os indicadores de desempenho foram: coeficiente de determinação $\mathrm{R}^{2}$ de 0,926 , índice de concordância de Willmott de 0,965 e erro absoluto médio (EAM) de $-0,186$. Valores similares aos encontrados por Bastos (1999), que obteve coeficientes de determinação variando de 0,77 a 0,98 e erros absolutos médios variando de 0,16 a 0,85 . Por outro lado, Bastos et al. (2012) encontraram valores máximos de IAF variando de 3,60 a 4,99 para a cultivar BRS Guariba no Piauí.

Com relação à produção de grãos, o valor observado (1.556 $\left.\mathrm{kg} \mathrm{ha}^{-1}\right)$ foi muito próximo do valor obtido na simulação ( $(1.572$ $\mathrm{kg} \mathrm{ha}^{-1}$ ), diferença de $1 \%$, compatível com os resultados de Bastos (1999) ao encontrar diferenças entre a produção de campo e a simulada variando de 3,8 a 14,9\%, trabalhando com as cultivares BR 14 - Mulato e BR - 17 Gurguéia. Oliveira et al. (2012) encontraram, em simulações com o CROPGROdrybean, diferença média de $6,2 \%$. Ao avaliar o modelo quanto à determinação das fases fenológicas, observaram-se ajustes razoáveis aos dados de campo (Tabelas 6A, 6B, 6C e 6D), com a maior diferença para o aparecimento da primeira flor e da primeira vagem de 6 dias, para o primeiro grão de 7 dias e para a maturidade fisiológica de 6 dias.

$\mathrm{O}$ cultivo $\mathrm{P}_{\text {MAR07 }}$ apresentou resultados discrepantes; já nos cultivos de janeiro as diferenças foram todas de 1 dia (Tabelas 6A, 6B e 6C) com exceção da maturidade, quando essas foram de 5 dias (Tabela 6D).

Para o cultivo $\mathrm{P}_{\mathrm{MAl1} 10}$, no que diz respeito ao aparecimento da primeira flor (Tabela 6A), o modelo simulou uma diferença de 2 dias (49 DAS), para o aparecimento da primeira vagem (Tabela 6B) e a diferença entre o valor simulado e o observado foi de 1 dia, 55 DAE e 54 DAE, respectivamente. Em relação ao aparecimento do primeiro grão (Tabela $6 \mathrm{C}$ ) observou-se diferença de 1 dia (60 DAS para o simulado e 59 DAS para o observado), assim como a mesma diferença foi obtida para a maturidade fisiológica (79 DAS para o simulado e 78 DAS para o observado).

No cultivo $\mathrm{P}_{\mathrm{ONI}}$, para o aparecimento da primeira flor, da primeira vagem e do primeiro grão, a diferença entre o simulado e o observado foi de 5 dias (Tabela 6A, B e C). Mesmo assim, o modelo representou antecipação da maturidade fisiológica (diferença de 1 dia) devido ao déficit hídrico (Tabela 6D), conhecido como mecanismo de escape à seca ou fuga à seca. Esta antecipação da maturidade, devida ao déficit hídrico, também foi descria por Nascimento et al. (2011) como uma estratégia da planta em fechar os estômatos para não perder água para o meio, aumentando a temperatura da folha e antecipando o processo de maturação quando se encontra em situação de déficit hídrico, como foi o caso do $\mathrm{P}_{\mathrm{ONI}}$, que foi cultivado numa época em que as temperaturas máximas estavam acima de $30^{\circ} \mathrm{C}$ e a cultura estava sob déficit hídrico (Tabela 4).

$\mathrm{O}$ cultivo $\mathrm{P}_{\text {JUL11 }}$ apresentou diferenças de 6 dias para o aparecimento da primeira flor (Tabela $6 \mathrm{~A}$ ) e da primeira vagem (Tabela 6B), diferenças de 7 dias para o aparecimento do primeiro grão (Tabela 6C) e no caso da maturidade fisiológica não houve diferença entre o simulado e o observado (Tabela 6D). Percebe-se, portanto, que os cultivos $\mathrm{P}_{\mathrm{ONI}}$ e $\mathrm{P}_{\mathrm{JUL} 11}$ apresentaram extremos de maturidade fisiológica, provavelmente em função
Tabela 6. Resultados para simulação de aparecimento da primeira flor (A), da primeira vagem $(B)$, do primeiro grão (C) e de maturidade fisiológica (D)

\begin{tabular}{|c|c|c|c|}
\hline \multirow{2}{*}{ Cultivo } & Simulado & Observado & Diferença \\
\hline & \multicolumn{3}{|c|}{ (dias) } \\
\hline \multicolumn{4}{|l|}{ A. } \\
\hline $\mathrm{P}_{\text {MR07 }}$ & 43 & 47 & -4 \\
\hline $\mathrm{P}_{\mathrm{J} 0}$ & 41 & 40 & 1 \\
\hline$P_{\mathrm{J} 25}$ & 41 & 40 & 1 \\
\hline $\mathrm{P}_{\mathrm{J} 75}$ & 41 & 40 & 1 \\
\hline $\mathrm{P}_{\mathrm{J} 100}$ & 41 & 40 & 1 \\
\hline $\mathrm{P}_{\text {MAl10 }}$ & 51 & 49 & 2 \\
\hline $\mathrm{P}_{\mathrm{ONI}}$ & 44 & 39 & 5 \\
\hline$P_{J U L 11}$ & 57 & 51 & 6 \\
\hline \multicolumn{4}{|l|}{ B. } \\
\hline $\mathrm{P}_{\mathrm{MR} 07}$ & 47 & 50 & -3 \\
\hline $\mathrm{P}_{\mathrm{J} O}$ & 44 & 43 & 1 \\
\hline$P_{\mathrm{J} 25}$ & 44 & 43 & 1 \\
\hline $\mathrm{P}_{\mathrm{J} 75}$ & 44 & 43 & 1 \\
\hline $\mathrm{P}_{\mathrm{J} 100}$ & 44 & 43 & 1 \\
\hline $\mathrm{P}_{\text {MAl10 }}$ & 55 & 54 & 1 \\
\hline $\mathrm{P}_{\mathrm{ONI}}$ & 47 & 42 & 5 \\
\hline$P_{\text {JUL11 }}$ & 60 & 54 & 6 \\
\hline \multicolumn{4}{|l|}{ C. } \\
\hline $\mathrm{P}_{\text {MR07 }}$ & 51 & 55 & -4 \\
\hline $\mathrm{P}_{\mathrm{JO}}$ & 48 & 47 & 1 \\
\hline $\mathrm{P}_{\mathrm{J} 25}$ & 48 & 47 & 1 \\
\hline$P_{\mathrm{J} 75}$ & 48 & 47 & 1 \\
\hline$P_{\mathrm{J} 100}$ & 48 & 47 & 1 \\
\hline $\mathrm{P}_{\text {MAl10 }}$ & 60 & 59 & 1 \\
\hline $\mathrm{P}_{O N I}$ & 51 & 46 & 5 \\
\hline$P_{\text {JUL11 }}$ & 65 & 58 & 7 \\
\hline \multicolumn{4}{|l|}{ D. } \\
\hline $\mathrm{P}_{\text {MR07 }}$ & 68 & 74 & -6 \\
\hline $\mathrm{P}_{\mathrm{JO}}$ & 64 & 69 & 5 \\
\hline$P_{\mathrm{J} 25}$ & 64 & 69 & 5 \\
\hline $\mathrm{P}_{\mathrm{J} 75}$ & 64 & 69 & 5 \\
\hline$P_{J 100}$ & 64 & 69 & 5 \\
\hline $\mathrm{P}_{\text {MAl10 }}$ & 79 & 78 & 1 \\
\hline $\mathrm{P}_{\mathrm{ONI}}$ & 64 & 63 & 1 \\
\hline$P_{J U L 11}$ & 83 & 83 & 0 \\
\hline
\end{tabular}

do déficit hídrico sofrido pelo $\mathrm{P}_{\mathrm{ONI}}$ (Tabela 4) e pelas baixas temperaturas ocorridas durante o cultivo $\mathrm{P}_{\mathrm{JUL} 11}$ (Figura 5E).

Comparando os resultados da fenologia da fase de avaliação com os da fase de calibração, percebem-se diferenças de seis e até sete dias mas, levando-se em conta que os experimentos das duas fases (calibração e avaliação) não foram submetidos às mesmas condições de água no solo e clima, tais resultados indicam uma boa performance do modelo.

De modo geral, percebe-se que os dados de campo e os simulados pelo modelo se aproximaram mais na fase vegetativa, na fase reprodutiva os dados se distanciaram, provavelmente por conta do aparecimento das flores e posteriormente das vagens na partição de foto assimilados tal como descrito por Wolschick et al. (2007), quando os autores incluíram, no modelo trabalhado, a translocação da matéria seca das folhas e caules para os grãos de milho melhorando os resultados simulados porém esta alteração não foi suficiente para acompanhar o aumento acelerado de matéria seca nos grãos.

Para o cultivo $\mathrm{P}_{\mathrm{MAR} 07}$ os indicadores foram EAM de $-512 \mathrm{~kg}$ ha $^{-1}, R^{2}$ de 0,95 e dW de 0,89 (Figura 3A). Os cultivos de janeiro (Figura 3B, C, D e E) com exceção do $\mathrm{P}_{\mathrm{J} 0}$, tiveram simulações próximas entre si porém muito distantes dos valores observados 

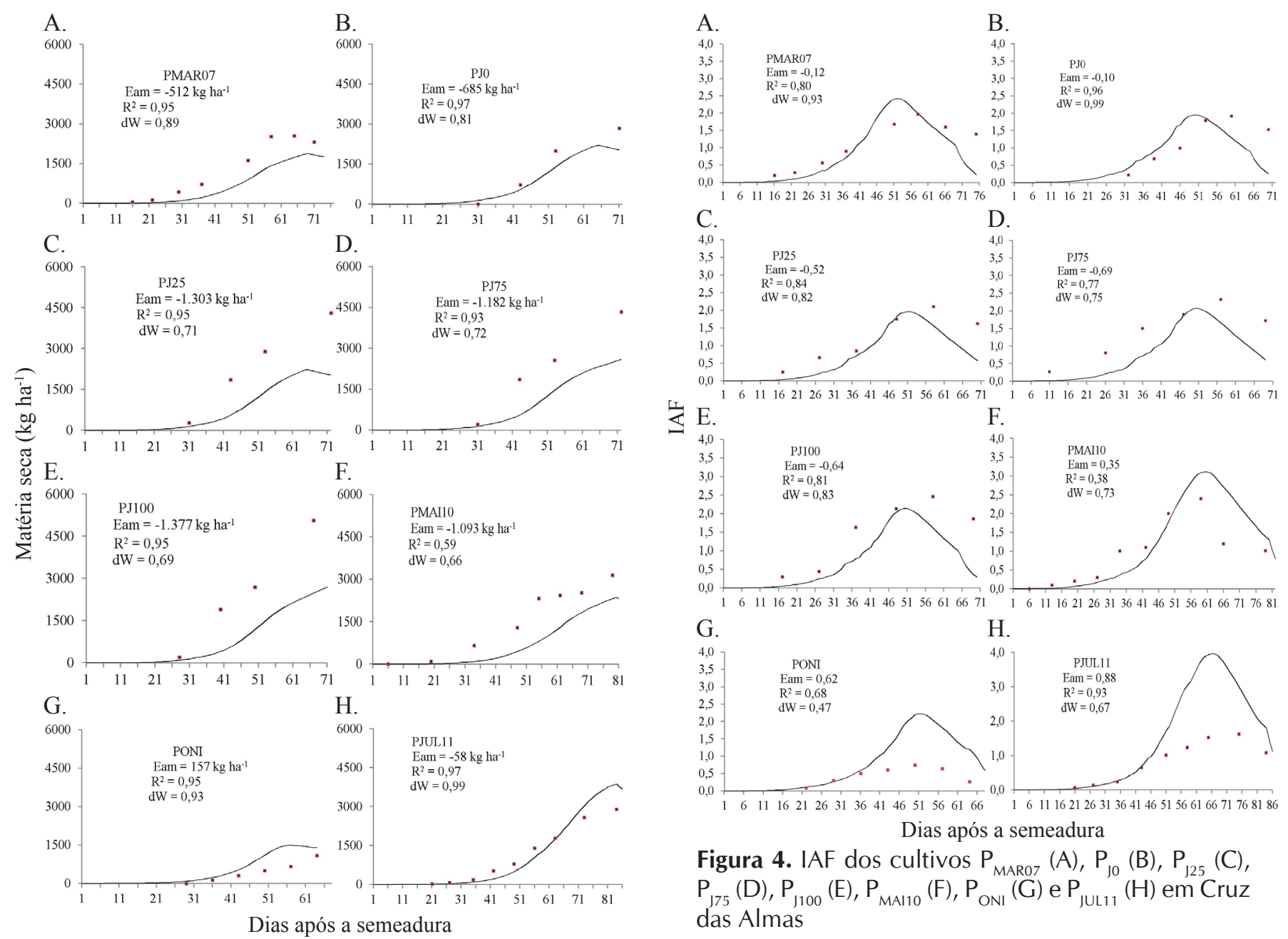

Figura 3. Estimativa de Matéria Seca dos cultivos

$$
\begin{aligned}
& P_{\text {MAR07 }}(A), P_{J 0}(B), P_{J 25}(C), P_{J 75}(D), P_{J 100}(E), P_{\text {MAI10 }}(F), \\
& P_{\text {ONI }}(G) \text { e } P_{J U L 11}(H)
\end{aligned}
$$

demonstrando pouca precisão do modelo, assim como o cultivo $\mathrm{P}_{\text {MAII0 }}$ (Figura 3F) que apresentou resultados discrepantes. Percebese, então, que o modelo não refletiu o ganho de matéria seca obtido nos cultivos. Os melhores resultados foram obtidos nas simulações dos cultivos $\mathrm{P}_{\mathrm{ONI}}$ e $\mathrm{P}_{\text {JUL11 }}$ (Figura $3 \mathrm{G}$ e $3 \mathrm{H}$, respectivamente).

No cultivo $\mathrm{P}_{\mathrm{ONI}}$ os resultados foram: EAM de $157 \mathrm{~kg} \mathrm{ha}^{-1}$, $\mathrm{R}^{2}$ de 0,95 e dW de 0,93 ; ja para o cultivo $\mathrm{P}_{\mathrm{JUL} 11}$ os indicadores foram EAM de $-58 \mathrm{~kg} \mathrm{ha}^{-1}, \mathrm{R}^{2}$ de 0,97 e dW de 0,99. Para os demais cultivos o erro absoluto médio foi alto, sobremaneira para os cultivos de janeiro e de maio, apesar de altos valores de $\mathrm{R}^{2}$, sugerindo que este indicador por si só não é suficiente para apontar os melhores resultados devendo-se recorrer ao índice de concordância de Willmott que, neste caso, apresenta valores mais baixos, concordando com o EAM.

O cultivo $\mathrm{P}_{\text {MAR07 }}$ (Figura 4A) teve seu IAF máximo estimado em 2,43 quando o valor de campo apontou 1,97. Dentre os cultivos de janeiro (Figura 4B, 4C, 4D e 4E) o $\mathrm{P}_{\mathrm{J} 0}$ apresentou os melhores resultados.

Os dados de IAF do cultivo $\mathrm{P}_{\text {MAII0 }}$ (Figura 4F) foram subestimados pelo modelo até aproximadamente 47 dias após a semeadura, daí em diante os dados são superestimados. O IAF máximo observado foi de 2,4 e o simulado, de 3,1. Para os cultivos $\mathrm{P}_{\mathrm{ONI}}$ e $\mathrm{P}_{\mathrm{JUL} 11}$ (Figura $3 \mathrm{G}$ e $4 \mathrm{H}$ respectivamente) os

Figura 4. IAF dos cultivos $\mathrm{P}_{\text {MAROZ }}(\mathrm{A}), \mathrm{P}_{10}(\mathrm{~B}), \mathrm{P}_{125}(\mathrm{C})$, $P_{J 75}(D), P_{J 100}(E), P_{\text {MAl10 }}(F), P_{\text {ONI }}(G)$ e $P_{J U L 11}(H)$ em CruZ das Almas

dados estimados foram muito superiores aos dados de campo, sendo o IAF máximo estimado em 2,23 e o máximo observado de 0,75 . O cultivo $\mathrm{P}_{\text {JUL11 }}$ teve, por sua vez, AF máximo estimado em 3,97 para um valor observado de 1,62.

Observando os indicadores de desempenho aplicados aos dados de IAF percebe-se que os melhores resultados são para o $\mathrm{P}_{\mathrm{J} 0}$, com um erro absoluto médio de $-0,10$ coeficiente de determinação de 0,96 e índice de concordância de Willmott de 0,98 . Para os demais de janeiro os índices foram razoáveis, com média de - 0,62 para EAM, 0,81 para $\mathrm{R}^{2}$ e 0,80 para dW. Para o cultivo $\mathrm{P}_{\text {MAR07 }}$ (Figura 4A) os índices de desempenho foram bons apresentando erro absoluto médio de $-0,12$, coeficiente de determinação de 0,80 e índice de concordância de Willmott de 0,93 . Para o cultivo com déficit hídrico $\left(\mathrm{P}_{\mathrm{ONI}}\right)$ os índices de desempenho não demonstraram bom resultado, pois foram encontrados: um erro absoluto médio de 0,62 , coeficiente de determinação de 0,68 e índice de concordância de Willmott de 0,47 , assim como para os índices de $\mathrm{P}_{\text {JUL11 }}$ os valores foram EAM de $0,88, R^{2}$ de 0,93 e dW de 0,66 .

O modelo subestimou a produtividade do cultivo $\mathrm{P}_{\mathrm{MR} 07}$ em 5,4\% (Tabela 7). Para os cultivos de janeiro as respostas foram menos precisas, as diferenças entre os valores simulados e observados foram de $-16,2,-20,1,-19,8$ e $-18,5 \%$ respectivamente para os cultivos $\mathrm{P}_{\mathrm{J} 0}, \mathrm{P}_{\mathrm{J} 25}, \mathrm{P}_{\mathrm{J} 75}$ e $\mathrm{P}_{\mathrm{J} 100}$, quando a média das temperaturas máximas no florescimento foi de $33,7^{\circ} \mathrm{C}$ (Figura 5B). 
Tabela 7. Produtividade de caupi observada e simulada em Cruz das Almas

\begin{tabular}{|c|c|c|c|c|}
\hline Cultivo & Simulado & Observado & Diferença & Diferença \\
\hline $\mathrm{P}_{\text {MR07 }}$ & 979,0 & $\begin{array}{l}1.035,0 \\
\end{array}$ & $-56,0$ & $-5,4$ \\
\hline $\mathrm{P}_{\mathrm{J} 0}$ & $1.139,0$ & $1.359,0$ & $-220,0$ & $-16,2$ \\
\hline $\mathrm{P}_{\mathrm{J} 25}$ & $1.147,0$ & $1.435,0$ & $-288,0$ & $-20,1$ \\
\hline $\mathrm{P}_{\mathrm{J} 75}$ & $1.174,0$ & $1.464,0$ & $-290,0$ & $-19,8$ \\
\hline $\mathrm{P}_{\mathrm{J} 100}$ & $1.197,0$ & $1.469,0$ & $-272,0$ & $-18,5$ \\
\hline $\mathrm{P}_{\text {Ma10 }}$ & $1.200,0$ & $1.058,0$ & 142,0 & 13,4 \\
\hline PONI & 399,0 & 576,0 & $-177,4$ & $-30,8$ \\
\hline PJUL11 & $1.603,0$ & $1.484,0$ & 119,0 & 8,0 \\
\hline
\end{tabular}

O feijão-caupi se desenvolve numa faixa de temperatura entre 20 e $35^{\circ} \mathrm{C}$, com faixa ideal de temperatura para a germinação da cultura de 23 a $32,5^{\circ} \mathrm{C}$ e a faixa de temperatura para a formação de nódulos entre $24 \mathrm{e} 33^{\circ} \mathrm{C}$. Altas temperaturas durante o período de florescimento reduzem o pegamento floral e prejudicam a floração enquanto baixas temperaturas prolongam o ciclo da planta (Bastos, 1999).

A média de produtividade observada para janeiro foi de $1.431,75 \mathrm{~kg} \mathrm{ha}^{-1}$, dentro da faixa esperada para o Nordeste, de modo geral pois, segundo Passos et al. (2011), nos últimos doze anos foram lançadas comercialmente 18 cultivares de caupi para a região sendo que as produtividades dessas cultivares em cultivo irrigado se situam na faixa de 1.500 a $2.000 \mathrm{~kg} \mathrm{ha}^{-1}$.

Meireles et al. (2002) encontraram, trabalhando com o modelo CROPGRO-drybean aplicado ao feijão carioca (Phaseolus vulgaris L.), respostas satisfatórias para a fenologia e a produtividade de grãos foi superestimada em, no máximo, $15,4 \%$, indicando o potencial do modelo para sua aplicação em Santo Antônio de Goiás, GO.

Com relação à produtividade do cultivo $\mathrm{P}_{\mathrm{MAII} 1}$, a diferença entre o valor simulado e o observado foi de $13,4 \%$. Neste cultivo as temperaturas mínimas também foram baixas (Figura 5C), prolongando o ciclo da cultura, com média de $19,5^{\circ} \mathrm{C}$ desde a floração até a colheita. A este respeito, Araújo \& Watt (1988), comentam que baixas temperaturas noturnas são o fator responsável pelo atraso na data de floração do caupi e retardamento do seu ciclo e prolongamento do crescimento, podendo ocorrer até completa inibição do florescimento em linhagens sensíveis.

Para a simulação da produtividade do cultivo $\mathrm{P}_{\mathrm{ONI}}$ houve subestimativa de $30,8 \%$ em relação à produtividade observada cujo modelo não simulou com precisão o déficit hídrico ocorrido (Tabelas 4 e 7). As temperaturas máximas foram, em média, $32{ }^{\circ} \mathrm{C}$ (Figura 5D) durante a floração; é provável, então, que o déficit hídrico tenha limitado o crescimento e a produção.

As simulações para o cultivo $\mathrm{P}_{\text {JUL11 }}$ apresentaram bom resultado, com diferença de apenas $8 \%$ em relação à produtividade observada. Neste cultivo não houve déficit hídrico (Tabelas $4 \mathrm{e} 7$ ) e foram registradas as temperaturas mais baixas (Figura 5E), média de temperaturas mínimas de $18,9^{\circ} \mathrm{C}$ desde a floração até a colheita o que, sem dúvida, prolongou o ciclo da cultura não comprometendo, no entanto, a produção, concordando parcialmente com Summerfield et al. (1985), os quais afirmam que temperaturas abaixo de $19{ }^{\circ} \mathrm{C}$ influenciam negativamente na produtividade do caupi, postergando o aparecimento das flores e aumentando o ciclo da cultura.
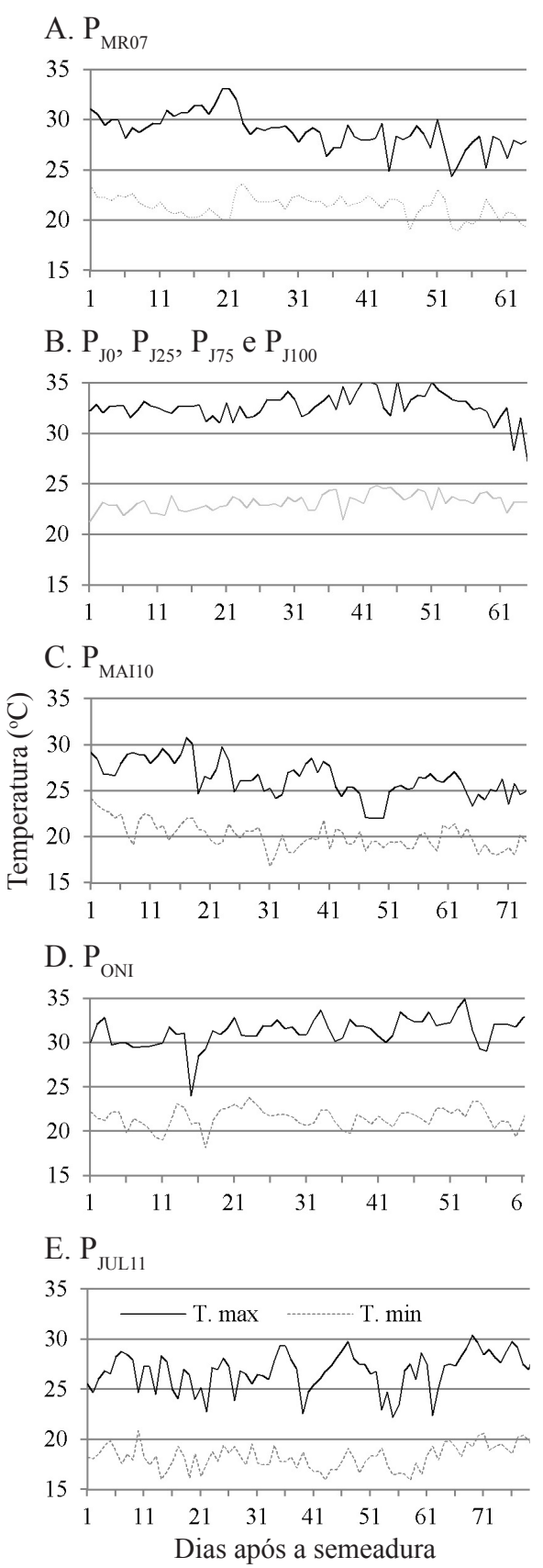

Figura 5. Valores das temperaturas máxima e mínima ocorridas durante os cultivos $\mathrm{P}_{\text {MAROT }}(\mathrm{A})$, de janeiro de 2010 (B), $P_{\text {MAI10 }}$ (C), $P_{\text {ONI }}$ (D) e $P_{\text {JUL11 }}$ (E) em Cruz das Almas

De modo geral, as respostas foram satisfatórias, principalmente se considerando que os cultivos foram implantados em diferentes condições de clima e água no solo indicando, portanto, boa eficiência do modelo.

\section{Conclusões}

1. O modelo foi ajustado aos dados de campo com boa precisão na fase de calibração; na fase de avaliação este simulou satisfatoriamente as fases fenológicas do feijão caupi nas condições do experimento.

2. O modelo simulou satisfatoriamente a produtividade e a matéria seca do caupi nas condições do experimento, com 
exceção para as condições de déficit de água no solo (cultivo $\mathrm{P}_{\mathrm{ONI}}$, de outubro de 2010).

\section{Literatura Citada}

Araújo, J. P. P.; Watt, E. E. (Org.) O caupi no Brasil. Brasília: Embrapa CNPAF, 1988, 722p.

Bastos, E. A. Adaptação do modelo CROPGRO para simulação do crescimento e desenvolvimento do feijão caupi (Vigna unguiculata (L.) Walp.) sob diferentes condições hídricas, no estado do Piauí. 91p. Piracicaba: ESALQ/USP, 1999. Tese Doutorado

Bastos, E. A.; Ferreira, V. M.; Silva, C. R. da; Andrade Júnior, A. S. de. Evapotranspiração e coeficiente de cultivo do feijãocaupi no Vale do Gurguéia, Piauí. Irriga, v.13, p.182-190, 2008.

Bastos, E. A, Ramos, H. M. M., Andrade Júnior, A. S., Nascimento, F. N., Cardoso, M. J. Parâmetros fisiológicos e produtividade de grãos verdes do feijão-caupi sob déficit hídrico. Water Resources and Irrigation Management, v.1, p.31-37, 2012.

Brasil. Ministério da Agricultura, Pecuária e Abastecimento. Secretaria de Defesa Agropecuária. Regras para análise de sementes. Brasília: MAPA/ACS, 2009. 399p.

Cruz, T. V.; Peixoto, C. P.; Martins, M. C. Crescimento e produtividade de soja em diferentes épocas de Semeadura no oeste da Bahia. Scientia Agraria, v.11, p.33-42, 2010.

Dallacort, R.; Freitas, P. S. L. de; Faria, R. T. de; Gonçalves, A. C. A.; Jácome, A. G.;Rezende, R. Soil water balance simulated by CROPGRO - Drybean model for edaphoclimatic conditions in Maringá. Revista Brasileira de Engenharia Agrícola e Ambiental, v.14, p.351-357, 2010.

Heinemann, A. B; Andrade, C. de L. T. de; Gomide, R. L.; Amorim, A. de O.; Paz, R. L. da. Padrões de deficiência hídrica para a cultura de milho (safra normal e safrinha) no estado de Goiás e suas conseqüencias para o melhoramento genético. Ciência e Agrotecnologia, v.33, p.1026-1036, 2009.

Heinemann, A. B.; Dingkuhn, M.; Luquet, D.; Combres, J. C.; Chapman, S. Characterization of drought stress environments for upland rice and maize in central Brazil. Euphytica, v.162, p.395-410, 2008.

Hoogenboom, G.; Jones, J. W.;Wilkens, P. W.; Porter, C. H.; Batchelor, W. D.; Hunt, L. A.; Boote, K. J.; Singh, U.; Uryasev, O.; Bowen, W. T.;Gijsman, A. J.; Du Toit, A. S.; White, J. W.; Tsuji, G. Y. Decision support system for agrotechnology transfer version 4.0. Honolulu: Universityof Hawaii, 2004. CD-Rom
Lima, C. J. G. S.; Oliveira, F. A.; Medeiros, J. F. de; Oliveira, M. K. T.; Oliveira Filho, A.F. Modelos matemáticos para estimativa de área foliar de feijão caupi. Revista Caatinga, v.21, p.120-127, 2008.

Lorençoni, R.; Dourado Neto, D.; Heinemann, A. B. Calibração e avaliação do modelo ORYZA-APSIM para o arroz de terras altas no Brasil. Revista Ciência Agronômica, v.41, p.605-613, 2010

Meireles, E. J. L.; Pereira, A. R.; Sentelhas, P. C.; Stone, L. F.; Zimmermann, F. J. P. Calibração e teste do modelo CROPGRO-drybean para as condições edafoclimáticas do Brasil Central. Scientia Agricola, v.59, p.723-729, 2002.

Muniz, L. C.;Viu, M. A. O.; Magnabosco, C. U.; Lopes, D. T. Modelagem e simulação da agropecuária. Pubvet, v.1, p.1982-1263, 2007.

Nascimento, S. P. do; Bastos, E. A.; Araújo, E. C. E.; Freire Filho, F. R.; Silva, E. M. da. Tolerância ao déficit hídrico em genótipos de feijão-caupi. Revista Brasileira de Engenharia Agrícola e Ambiental, v.15,p.853-860, 2011.

Oliveira, E. C. de; Costa, J. M. N. da; Paula Júnior, T. J. de; Ferreira, W. P. M.; Justino, F. B. J., Neves, L. de O. Desempenho do modelo CROPGRO-Drybean na simulação do rendimento de feijão (Phaseolus vulgaris L.). Acta Scientarium, v.34, p.239-246, 2012.

Passos, A. R.; Silva, S. A.; Peixoto, C. P.; Rocha, M. A. C. da; Cruz, E. M. de O. Ganho por seleção direta e indireta em caupi considerando a interação G x E. Revista da FZVA, v.18, p.18-33, 2011.

Sousa, M. A.; Lima, M. D. B.; Silva, M. V. V.; Andrade, J. W. S. Estresse hídrico e profundidade de incorporação do adubo afetando os componentes de rendimento do feijoeiro. Pesquisa Agropecuária Tropical, v.39, p.175$182,2009$.

Summerfield, D. J.; Pate, J. S.; Roberts, E. H.; Wien, H. C.The physiology cowpea. In: Singh, S. R.; Rachie, K.O. (ed.). Cowpea research, production and utilization. Chichester: John Wiley, p.66-101, 1985.

Wolschick, D.; Martinez, M. A.; Fontes, P. C. R.; Matos, A. T. de Implementação e teste de um modelo mecanístico de simulação do crescimento e desenvolvimento de plantas de milho. Revista Brasileira de Engenharia Agrícola e Ambiental, v.11, p.271-278, 2007. 